\title{
Entre desserte (péri)urbaine et desserte aéroportuaire, les tramways de l'Est lyonnais
}

The hard development of the public transport service to the airport and to the urban area. The case of the East Lyon tramway

Jean Varlet

\section{CpenEdition}

\section{Journals}

Édition électronique

URL : http://journals.openedition.org/rge/3598

DOI : $10.4000 /$ rge.3598

ISSN : $2108-6478$

Éditeur

Association des géographes de l'Est

Édition imprimée

Date de publication : 15 juin 2012

ISSN : 0035-3213

\section{Référence électronique}

Jean Varlet, "Entre desserte (péri)urbaine et desserte aéroportuaire, les tramways de l'Est lyonnais », Revue Géographique de l'Est [En ligne], vol. 52 / 1-2 | 2012, mis en ligne le 18 octobre 2012, consulté le 08 septembre 2020. URL : http://journals.openedition.org/rge/3598 ; DOI : https://doi.org/10.4000/ rge.3598

Ce document a été généré automatiquement le 8 septembre 2020

Tous droits réservés 


\section{Entre desserte (péri)urbaine et desserte aéroportuaire, les tramways de l'Est lyonnais}

The hard development of the public transport service to the airport and to the urban area. The case of the East Lyon tramway

Jean Varlet

1 Dans la période récente d'expansion de nouveaux réseaux de transports urbains lourds, les territoires concernés se trouvent évidemment aux premières loges, lorsqu'ils tentent de traiter en partie les problèmes de congestion automobile, de stationnement, de pollution et de financement des transports en commun afin de faciliter les déplacements des citadins, des banlieusards et des habitants du périurbain. Tout en étant soutenus par les récentes évolutions législatives (LAURE ${ }^{1}$ en 1996, SRU ${ }^{2}$ en 2000, Grenelle de l'Environnement ${ }^{3}$ en 2009), les acteurs publics locaux doivent faire face à des choix difficiles sur les plans stratégique et technique. Ils ont à se prononcer sur des types de dessertes, des modes de transport et des modalités de financement. Les options prises engagent sur le très long terme, à la fois les collectivités dans leurs politiques et les populations dans leurs pratiques de déplacement. Dès lors, quelle option choisir en matière de mode de transport, de tracé, de desserte des territoires? quels facteurs interviennent dans la prise de décision? Quelle place donner aux Transports en Commun en Site Propre pour réduire le poids de l'automobile sans toutefois obérer les déplacements nécessaires de toute une population dans les divers secteurs d'un territoire? Quelle option choisir entre le développement d'un réseau moderne de métro, ou de tramway selon la taille de l'agglomération, ou l'essor d'un réseau d'autobus ou encore la combinaison de plusieurs formules?

2 Le cas de l'Est lyonnais est emblématique à cet égard. Il constitue un exemple à la fois innovant et révélateur des divergences d'option dans l'avancement d'un processus, ainsi qu'un exemple de la complexité géographique d'un aménagement. Le tramway Rhônexpress mis en service en 2010 semble achever pour l'instant ce processus ; reliant désormais la gare de Lyon-Part-Dieu à la gare TGV de l'aéroport de Lyon-Saint- 
Exupéry, il emprunte une partie de l'ancienne voie ferrée des Chemins de Fer de l'Est Lyonnais, modernisée pour la circonstance, sur laquelle circule aussi et partiellement le tramway de la ligne T3 des Transports en Commun Lyonnais. L'un comme l'autre correspondent aux aboutissements d'un long processus de réflexion sur l'amélioration de la desserte en transports en commun d'une part de l'aéroport et d'autre part d'une banlieue puis d'un espace périurbain, c'est-à-dire de deux lieux et milieux à offres de transport bien spécifiques et à options divergentes puis conciliées.

3 C'est pourquoi l'analyse commence par présenter les modalités de l'organisation de la desserte des territoires sur ce nouvel axe lourd, avant d'en décortiquer le processus de réalisation puis le processus d'intégration dans le système de transport et d'aménagement de l'espace urbain et dans le système européen des dessertes aéroportuaires.

\section{Les lignes et territoires desservis}

4 Le caractère original et innovant de la nouvelle offre de transports collectifs mise en place sur l'axe Lyon-Part-Dieu - Lyon-Saint-Exupéry fait même de cette ligne un pionnier en France en la matière. Son fonctionnement et son dispositif institutionnels n'en demeurent pas moins complexes.

\section{A. Une ligne supportant deux dessertes spécifiques}

5 Quelle originalité et quelle innovation dans le contexte récent français que la mise en service progressive de ces tramways de l'Est lyonnais! En effet, sur une seule et même ligne reliant la Part-Dieu à Crémieu via Meyzieu circulent deux types de tramways qui desservent dans des conditions bien distinctes à la fois des espaces géographiques contrastés - un centre-ville, des banlieues, des milieux périurbains et un aéroport - et plusieurs échelles de mobilités - locale, régionale, nationale et internationale (Doc.1). 
Doc.1 - A la station "La Soie » : à droite, le tramway T3,et à gauche, le tramway Rhônexpress (Cliché J. Varlet, 9/09/2011)

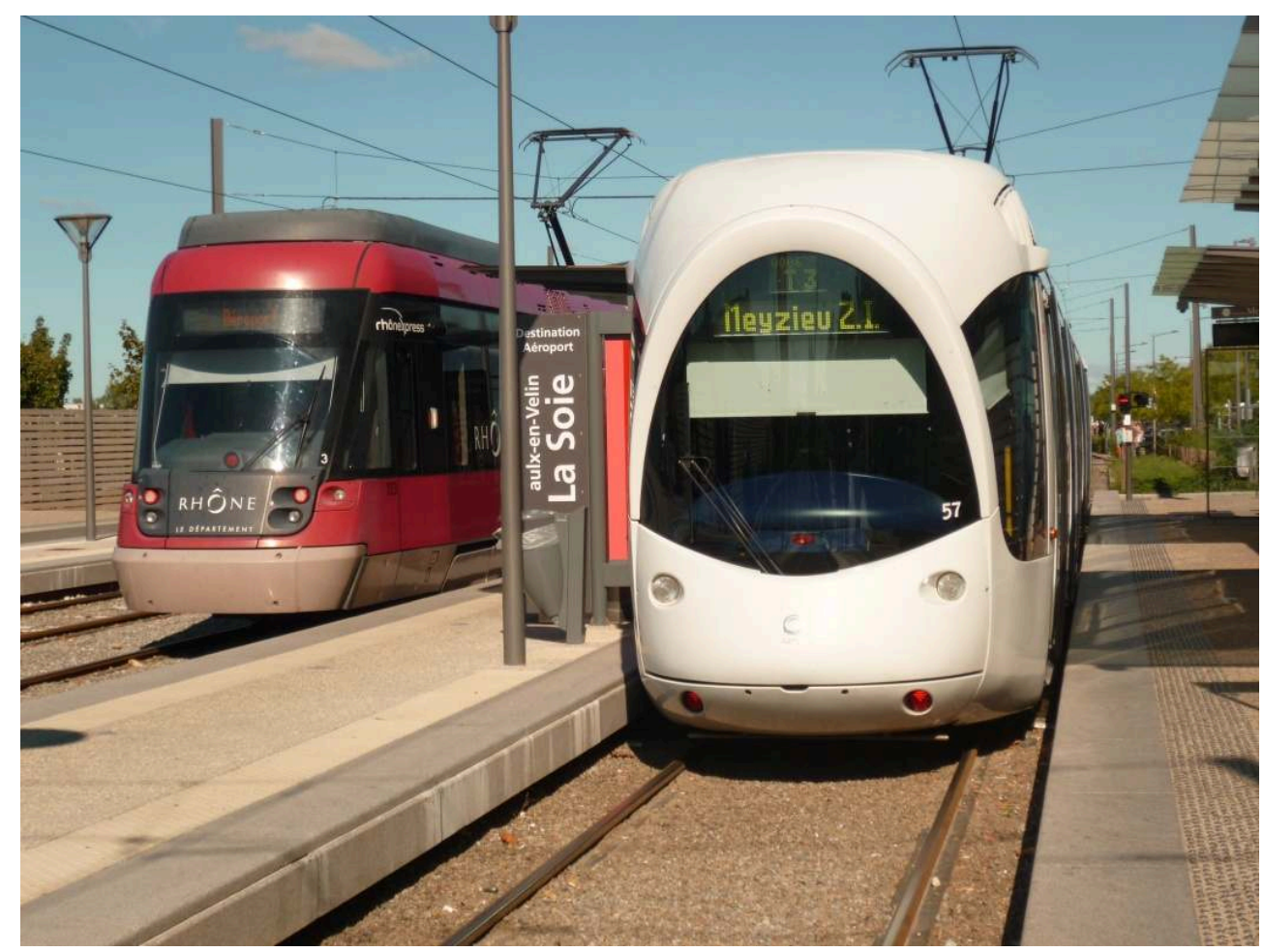

\section{Un tramway urbain classique}

7 Le 4 décembre 2006, la ligne T3 du tramway lyonnais est mise en service, jusque-là connue sous l'appellation $L E A$ en tant que projet d'aménagement d'un axe de transports lourds en milieu urbain ("Ligne de l'Est de l'Agglomération»). A une fréquence de 7', elle dessert dix stations égrenées de la gare centrale de La Part-Dieu à la station périurbaine de Meyzieu ZI via les banlieues du secteur de Vaulx-en-Velin (Doc.2a). Ces deux stations situées aux extrémités étant distantes de $14,6 \mathrm{~km}$, la distance moyenne interstations s'établit à $1,6 \mathrm{~km}$ ce qui constitue une valeur élevée, triple de la moyenne habituelle. Avec un parcours effectué en $25^{\prime}$, c'est là aussi une vitesse moyenne considérable qui est enregistrée : $38 \mathrm{~km} / \mathrm{h}$ au lieu des 17-18 habituels.

Exploitée par l'opérateur urbain public, TCL («Transports en Commun Lyonnais»), la ligne T3 offre une tarification urbaine qui donne droit de circuler sur tout le parcours avec un ticket urbain valable une heure (1,60 € en septembre 2011). Une moyenne de 24 000 voyageurs/jour est enregistrée en 2011, dans ce qui relève exclusivement de déplacements urbains, banlieusards et périurbains qui combinent éventuellement une complémentarité entre tramway et un autre transport collectif (autobus, métro) ou un véhicule individuel (vélo, automobile). 
Doc.2 - Les tramways de l'Est lyonnais : la complexité du dispositif.
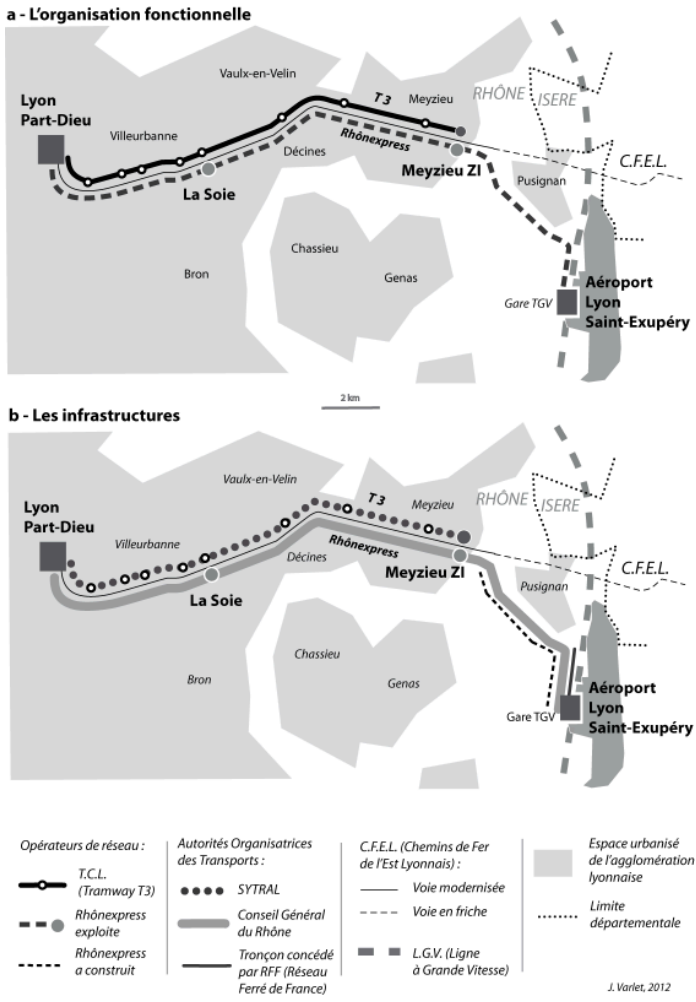

Doc.3 - Dans la plaquette promotionnelle du Rhônexpress.

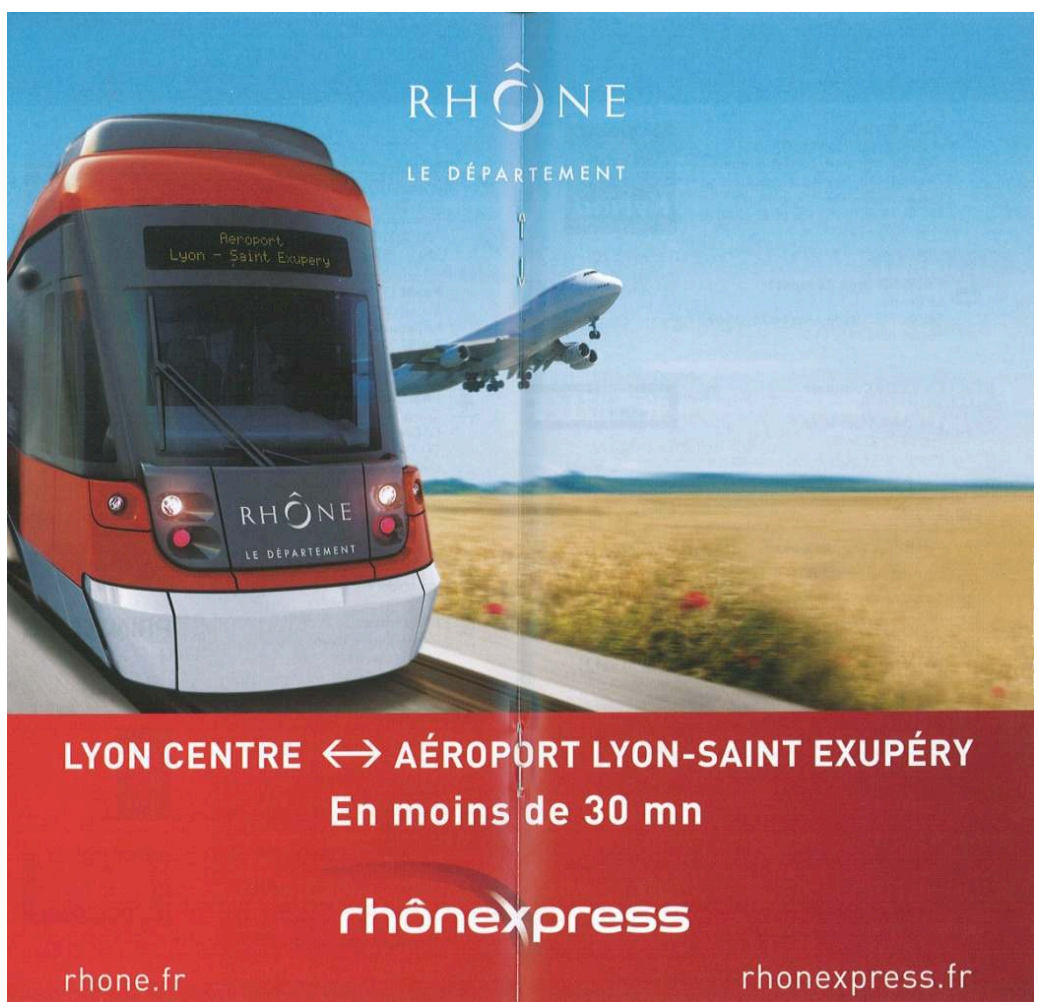

Un tramway express 
Depuis le 9 août 2010 circule aussi sur cette même ligne de chemin de fer un deuxième type de tramway, le Rhônexpress, qui va toutefois un peu plus loin, jusqu'à l'aéroport de Lyon - Saint-Exupéry, soit $7 \mathrm{~km}$ de plus (Doc.2a). Par comparaison et du fait de sa spécificité de relier Lyon à son aéroport, ses caractéristiques diffèrent sur de nombreux points :

- Ce tramway aux formes et au gabarit différents de ceux du T3 ne dessert que quatre stations : les deux extrémités, Lyon-Part-Dieu et Aéroport Lyon-Saint-Exupéry, et deux stations intermédiaires: La Soie et Meyzieu ZI. Ayant une obligation contractuelle d'effectuer le trajet en moins de 30' (Doc.3), les six rames disponibles de ce tramway (76 places chacune) parcourent ces $23 \mathrm{~km}$ à une vitesse moyenne de $47 \mathrm{~km} / \mathrm{h}$ (!), quasi-identique à celle de trains régionaux omnibus. Il atteint même les $100 \mathrm{~km} / \mathrm{h}$ sur les sept derniers kilomètres en rase campagne entre Meyzieu ZI et l'aéroport. Une fréquence au quart d'heure est assurée entre 6 ho0 et 21 ho0, à la demi-heure entre 5 h00 et 6 h00 ainsi qu'entre 21 hoo et $24 \mathrm{~h} 00$.

- L'exploitant est un opérateur privé, constitué pour la circonstance, dont le nom de Rhônexpress désigne aussi bien la compagnie que le tramway.

- Une tarification spécifique est volontairement adaptée à sa vocation et à sa clientèle. Fournissant une offre haut de gamme (ligne «Premium ») pour une clientèle aéroportuaire (affaires, tourisme, employés), elle se veut dissuasive pour les populations se déplaçant uniquement dans l'espace urbain sans se rendre à/depuis l'aéroport. D'où un tarif unique de $13 €$ l'aller simple ( $23 €$ l'aller-retour) quelles que soient les stations de départ et d'arrivée, c'est-à-dire un tarif plus élevé que celui des précédentes navettes autocars Satobus supprimées de facto dès la mise en service du Rhônexpress - établi à $9 €$ l'aller simple, et bien moins élevé que celui du taxi (50€ environ l'aller simple). Et avec un avantage incomparable: un gain de temps et une garantie de temps de parcours (moins de trente minutes) que la congestion automobile aux entrées/sorties de Lyon et en ville ne permettait pas.

- La compagnie Rhônexpress ambitionne d'atteindre un trafic d'un million de passagers au début - valeur en fait obtenue au bout de onze mois, en juillet 2011 - c'est-à-dire une moyenne de 2500 à 3000 voyageurs/jour, qu'elle espère porter à 4000 d'ici quelques années.

11 Enfin et bien que secondaire dans la présente approche, un troisième type de circulation coexiste parallèlement à celle du tramway: une piste cyclable, bidirectionnelle et éclairée la nuit, qui a été aménagée de la Part-Dieu à Meyzieu en longeant les voies sur dix kilomètres.

\section{B. La complexité du dispositif institutionnel}

Sous-jacente à ces caractéristiques d'organisation et de fonctionnement, la répartition des fonctions entre acteurs s'avère complexe et révèle la nature des enjeux le long et autour d'un axe redevenu majeur.

\section{Une infrastructure locale ancienne}

Le support aux circulations actuelles est l'ancienne voie ferrée des CFEL qui relia Lyon à Crémieu (environ $40 \mathrm{~km}$ ) et qui fonctionna de 1881 à 1947, date à laquelle fut arrêté le trafic voyageurs (Doc.2). L'essor du trafic fret nécessita un raccordement ferroviaire au niveau de la gare actuelle de la Part-Dieu (1950) avant que s'amorce un déclin à partir de 1968, avant que le terminus soit ramené de Crémieu à Meyzieu (1978) et avant que la 
SNCF cesse l'exploitation de la ligne (1999). D'un million de tonnes en 1969, le trafic fret est passé à 150000 T/an dans les décennies 1980 et 1990, soit deux trains par jour.

Les deux Départements du Rhône et de l'Isère, propriétaires de la ligne depuis 1977, durent compenser les pertes, mais le retrait du Conseil général de l'Isère laissa seul le Conseil général du Rhône.

\section{Une ligne reconsidérée et réappropriée}

Présentement, cette ancienne infrastructure est désormais méconnaissable. Si le tracé et l'emprise ont été conservés, des modifications majeures ont été apportées.

Seul le tronçon Part-Dieu - Meyzieu ZI a été remis en service avec une infrastructure totalement rénovée, le segment situé plus à l'Est étant laissé en friche (Doc.2b). D'une voie unique, il est passé à deux voies; et à une voie à traction thermique s'est substituée une voie électrifiée.

Entre Meyzieu ZI et l'aéroport, un nouveau tracé a été construit (Doc.4) : plutôt que de réaliser un raccord à angle droit entre la ligne des CFEL et la LGV juste à l'Est de Pusignan - solution logique proposée de longue date -, un tracé plus court a été préféré privilégiant l'hypoténuse sur un peu plus de six kilomètres puis un segment parallèle à la LGV sur 700 mètres. Ce choix de tracé, plus court et à vitesse élevée (traction électrique), permet d'une part de tenir l'obligation inscrite dans le cahier des charges d'un trajet aéroport-centre-ville en moins de trente minutes, et d'autre part de bénéficier d'un coût du foncier moins élevé en traversant les champs plutôt que l'agglomération de Pusignan tout en évitant un problème de largeur insuffisante de l'emprise dans cette dernière traversée.

Dans le cadre d'un appel d'offres concurrentiel pour l'attribution de la concession, et bien que se faisant au détriment de la notion de moindre consommation d'espace, cette proposition de tracé permettant de respecter les obligations du cahier des charges fit emporter le marché au groupement Rhônexpress. 
Doc.4 - A l'Est de la station « Meyzieu ZI » : à gauche, l'ancienne voie CFEL en direction de Pusignan), et à droite, la nouvelle voie Rhônexpress (double voie électrifiée) en direction de l'aéroport (Cliché J. Varlet, 9/09/2011)

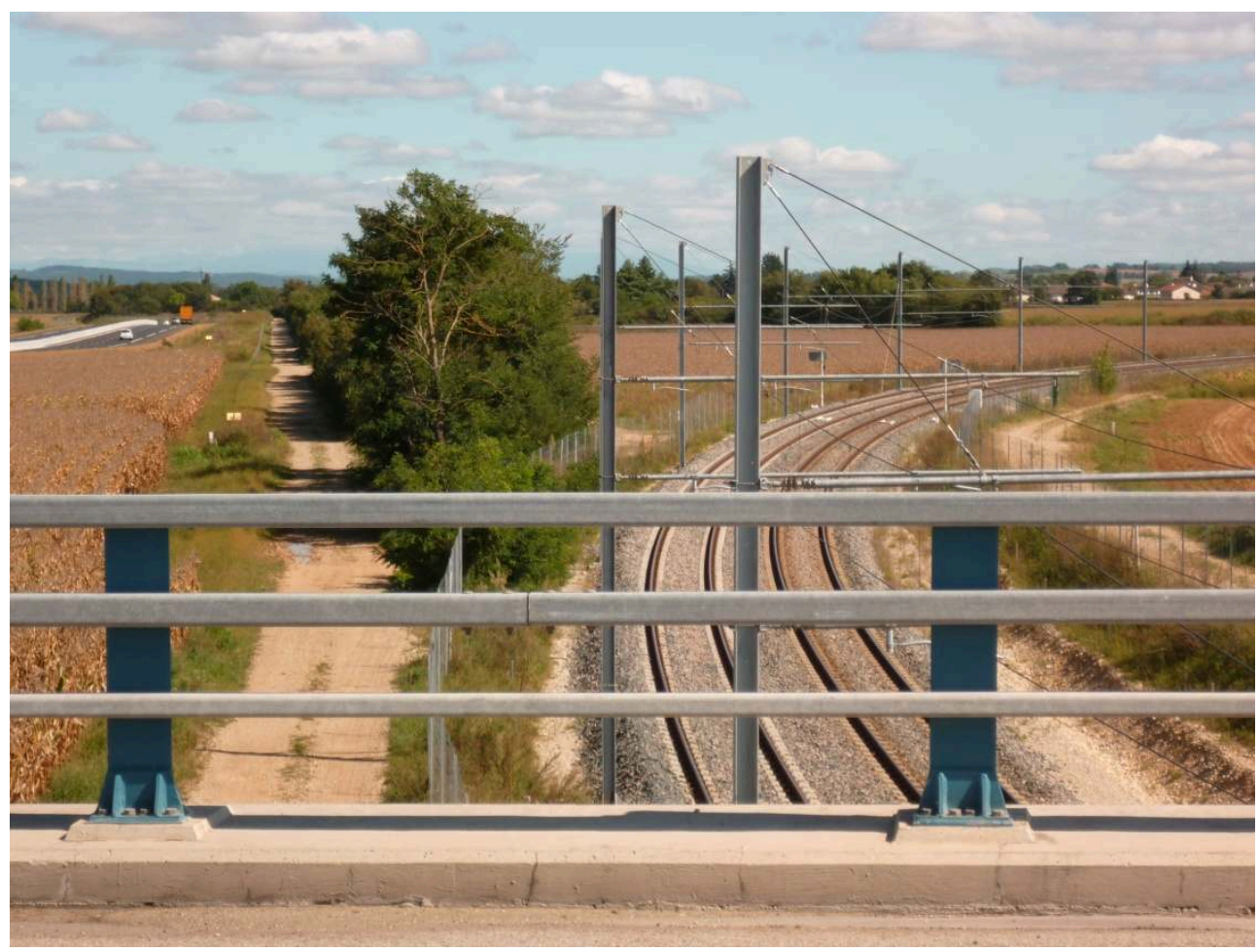

21 En matière de réseau-support (infrastructures), trois segments sont donc à différencier quant aux acteurs responsables :

- De Lyon-Part-Dieu à Meyzieu ZI, le SYTRAL (Syndicat Mixte des Transports pour le Rhône et l'Agglomération Lyonnaise), АOTU ${ }^{4}$ dont sont membres le Grand Lyon et le Conseil général du Rhône, a pris en charge la remise en état de l'emprise ferroviaire, autrement dit les dessertes urbaine et périurbaine. Le Conseil général a mis à disposition la ligne dont il est propriétaire.

- Entre Meyzieu ZI et la gare TGV de l'aéroport, le Conseil général du Rhône, autorité organisatrice entre Lyon-Part-Dieu et l'aéroport, a concédé la construction et l'exploitation de la ligne à la société Rhônexpress.

- En outre, sur le court segment longeant la LGV juste avant l'arrivée à la gare TGV, c'est RFF (Réseau Ferré de France) qui a accordé un contrat de concession à Rhônexpress.

En matière de réseau fonctionnel, deux compagnies différentes exploitent le tramway. La compagnie TCL (Transports en Commun Lyonnais), membre du groupe Keolis, s'est vue confier par le SYTRAL l'exploitation de la ligne T3, ajoutant ainsi un rayon supplémentaire à son réseau de tramways urbains. Tandis que l'exploitation de la desserte aéroportuaire depuis Lyon a été accordée par le Conseil général du Rhône à la société Rhônexpress, dont les trois actionnaires sont la Caisse des Dépôts et Consignations $(36,6 \%)$ pour l'investissement, Vinci $(35,2 \%)$ pour la construction, et Veolia Transport $(28,2 \%)$ pour l'exploitation.

23 L'infrastructure est ainsi confiée à deux opérateurs de réseau différents qui doivent faire fonctionner leurs réseaux en bonne entente, sur la base d'un cahier des charges bien précis, notamment sur deux points. D'une part la succession coordonnée des tramways sur la ligne selon le schéma suivant : un tramway Rhônexpress (fréquence de 
15') doit partir de La Part-Dieu juste avant une rame T3 (fréquence de 7') et arriver à Meyzieu ZI juste après la rame T3 précédente. D’autre part, au poste de commandement, commun, la gestion de l'ensemble est assurée par le SYTRAL, ce qui place les rames Veolia sous « contrôle » de Keolis.

Ainsi est-on en présence pour la première fois en France d'un système qui, sur une même voie ferrée, combine en étroites collaboration et entente: deux types de dessertes (urbaine/périurbaine et aéroportuaire), deux types bien différents de clientèles, deux AOT et deux types d'opérateurs de réseau, l'un public et l'autre privé (première ligne ferroviaire voyageurs concédée au secteur privé en France dans la période récente).

Dans la mesure où ce système fonctionne en mode fermé entre les deux extrémités, avec des tramways classiques, périurbains au plus jusqu'à un aéroport, l'appellation tram-train ne peut pas convenir puisque, même si ces tramways empruntent une ancienne voie ferrée autrefois réservée à des trains, aujourd'hui totalement absents, aucun de ces tramways ne prolonge son parcours sur le réseau ferroviaire classique et n'y circule en alternance avec des trains.

\section{D'un projet a un autre : quatre décennies d'hésitations des territoires}

Eu égard aux répartitions de compétences et alors même que les cadre ferroviaires et territoriaux sont marqués par tant de rigidités et induisent tant de discontinuités, comment est-on parvenu à organiser un tel dispositif?

Tandis qu'une desserte ferroviaire des aéroports est enclenchée dans les métropoles européennes dans la décennie 1980 (Zürich, Amsterdam, Francfort, Genève), avant de se généraliser dans les décennies suivantes (Varlet, 1992 et 2000), l'agglomération lyonnaise, tout intéressée qu'elle est par le principe, reste longtemps prudente et indécise, avant de s'engager dans le processus qui aboutit à la mise en place du système désormais en service. Si la desserte de l'aéroport est bien le déclencheur de la réflexion et des évolutions ultérieures, la desserte d'une agglomération en extension prend plus tard le relais.

28 Au cœur de cette succession de deux périodes revient en permanence le problème de la mixité des fonctions et vocations sur une seule et même ligne (Mathurin, 2002), considéré d'abord comme insoluble, dans un premier contexte au regard des coûts induits et des aspects techniques des dessertes, puis comme résolu dans un deuxième contexte. Après s'être annulées, les options s'additionnent.

\section{A. Une phase d'opposition de deux logiques inconciliables}

A posteriori il ressort bien une première période où émergent des options différentes, progressivement émises par des acteurs territoriaux aux intérêts et compétences distincts, et où se manifestent de longues tergiversations au sein desquelles les problèmes de desserte, de modes de transport et de tracés se posent avec une réelle acuité.

Les tenants de la desserte aéroportuaire 
Chronologiquement, c'est d'abord le problème de la desserte aéroportuaire qui est soulevé, et deux acteurs principaux s'en font les promoteurs.

Fort logiquement, le concessionnaire de l'aéroport, la Chambre de Commerce et d'Industrie de Lyon, alerte les autorités dès 1973 sur le problème de l'accès à l'aéroport de Lyon, par suite de son transfert à une distance encore plus éloignée de l'agglomération : $25 \mathrm{~km}$ (Satolas) au lieu de $7 \mathrm{~km}$ jusqu'alors (Bron). Certes, l'autoroute A43 en direction de Chambéry et de Grenoble est lancée et une antenne vers l'aéroport est prévue, mais en termes de transports en commun la question reste posée. Trois solutions sont alors envisagées : soit une desserte par autocars, cadencés à la demiheure et au temps de parcours estimé à $35^{\prime}$ en heures creuses et à 55' en heures de pointe ; soit une desserte ferroviaire en $27^{\prime}$, qui associerait d'une part la ligne de Lyon à Grenoble, une antenne vers l'aéroport (à construire) jusqu'à une gare ferroviaire proche de l'aéroport (à créer) et d'autre part un parcours terminal en autobus en raison d'une distance trop grande entre la gare et l'aérogare; soit enfin, une liaison mixte de type "Orlyrail» utilisant successivement ladite voie ferrée jusqu'à Heyrieux puis l'autocar jusqu'à Satolas (44'). Pendant les décennies suivantes, la CCI de Lyon soulève régulièrement ce problème et reste l'acteur fondamental de cette option.

Plus tard, la Région Rhône-Alpes affirme sa volonté de favoriser la plate-forme aéroportuaire de Lyon, de lui donner une vocation régionale en la dotant d'un pôle d'échanges régional autour de l'aérogare et d'une gare ferroviaire. Option qui se concrétisera par la construction de cette monumentale et symbolique gare TGV, dans le double contexte d'extension $\mathrm{du}$ réseau ferré à grande vitesse contournant l'agglomération lyonnaise et d'attribution des Jeux Olympiques à Albertville en 1992. Dès 1989, la Région avait déjà acté un projet de desserte ferroviaire de l'aéroport de Satolas depuis le centre de Lyon, en 17' et trois fois par heure, en empruntant successivement la voie du CFEL jusqu'à Pusignan, puis une voie à créer vers le sud via l'aéroport pour rejoindre la " ligne du sud » Lyon-Grenoble au niveau de Saint-QuentinFallavier (Doc.5). En cela, elle reprenait partiellement le projet Satorail, apparu en 1980, qui prônait la réutilisation de la ligne CFEL comme ligne de banlieue et comme ligne « intercités » reliant Lyon aux villes de Savoie et du Dauphiné par l'aéroport de Satolas. $\mathrm{Au}$ cours des décennies, la Région paraît de moins en moins partie prenante du processus, bien qu'étant l'AOT hors périmètre urbain. Au bout du compte, le devenir de la desserte aéroportuaire confirme bien l'inversion dans les objectifs initiaux, lesquels auraient dû privilégier d'abord une gare régionale et un accès à la ville avant d'implanter une gare TGV (Plassard, 1990).

4 Dans ce type de desserte aéroportuaire, l'offre d'une liaison rapide et directe entre les deux extrémités, avec un nombre d'arrêts intermédiaires soit nul soit limité à deux ou trois, et avec un confort adapté à une clientèle aérienne encore majoritairement à haute contribution priment d'abord et avant tout.

Mais l'intention de relier l'aéroport au centre-ville par chemin de fer se heurte dès le début au refus catégorique de l'opérateur ferroviaire national, la SNCF, qui le justifie par l'absence d'un trafic d'au moins 4 millions de passagers aériens à Satolas (3,7 Mp en 1990).

\section{Les tenants d'une desserte périurbaine}

Parallèlement ou presque, les acteurs urbains interviennent dans la réflexion et dans le processus à partir de 1978, notamment l'AOTU : le syndicat des Transports en Commun 
de la Région Lyonnaise, devenu plus tard le SYTRAL, qui intervient régulièrement dans le débat pour prôner une autre approche.

Le Syndicat Mixte des Transports pour le Rhône et l'Agglomération Lyonnaise songe lui aussi à une réutilisation de la ligne du CFEL mais davantage pour assurer, jusqu'à Meyzieu, du trafic voyageurs à destination ou en provenance des communes de l'Est lyonnais, en forte croissance où est entamé un processus de développement périurbain. L'aéroport n'est donc pas sa préoccupation première au contraire de son objectif primordial d'une meilleure connexion des banlieues orientales avec le centre de l'agglomération lyonnaise. L'axe du CFEL existe et peut se révéler être une opportunité à saisir, en tant qu'infrastructure ferroviaire ou en tant qu'emprise foncière disponible pour y établir un mode de transport ou un autre; a contrario, la position de cette emprise dans l'espace urbain est plutôt défavorable du fait d'un tracé par trop éloigné des lieux d'habitat.

On comprend aisément que les collectivités territoriales de l'Est lyonnais aient appuyé l'idée d'une meilleure desserte en transports en commun de leur secteur, sans nécessairement prendre en compte le CFEL.

Nombreux auront été les projets de desserte, les itinéraires, les modes envisagés, les technologies, les acteurs promoteurs... (Doc.5) : par le nord, le centre ou le sud; en train, en autocar, en autobus, en tramway, ou en solution combinée ; sur voie existante ou sur infrastructure à créer; par telle ou telle collectivité territoriale ou par telle association.

\section{B. Une phase de combinaison des deux logiques}

41 A ces deux décennies de prise de conscience, de recherche de formules appropriées, de balancement entre desserte de l'aéroport ou desserte urbaine et périurbaine, fait suite, à partir de la fin de la décennie 1990, une phase de combinaison des deux logiques qui apparaissait jusqu'alors comme incompatibles. Elle est le fruit de l'évolution et de la conjonction de plusieurs paramètres qui modifient progressivement le contexte et la faisabilité de tel ou tel projet.

\section{L'évolution des contextes}

Dans le registre des évolutions lentes, trois faits vont contribuer à faire progresser les idées. 
Doc.5a - Historique des projets de liaison Lyon - Satolas/Saint-Exupéry (tableau)

\begin{tabular}{|c|c|c|c|c|c|}
\hline Année & $\begin{array}{l}\text { Projet ou chargé } \\
\text { d'étude }\end{array}$ & Type de desserte & $\begin{array}{l}\text { Temps } \\
\text { estimé }\end{array}$ & $\begin{array}{l}\text { Mixité des } \\
\text { fonctions }\end{array}$ & $\begin{array}{l}\text { Cause } \\
\text { de non- } \\
\text { réalisation du } \\
\text { projet }\end{array}$ \\
\hline 1973 & & $\begin{array}{l}\text { Autocar } \\
\text { Train } \\
\text { Mixte train + car }\end{array}$ & $\begin{array}{l}35^{\prime} \\
27 \\
44^{\prime}\end{array}$ & $\begin{array}{l}\text { Aéroport } \\
\text { Aéroport } \\
\text { Aéroport }\end{array}$ & $\begin{array}{c}\text { Adopté } \\
\text { Surdimensionné } \\
\text { Coût et techniques }\end{array}$ \\
\hline & & Aérotrain & & & Coût et techniques \\
\hline 1978 & SYTRAL, SEMALY & $\begin{array}{l}\text { Prolongement métro } \\
\text { et CFEL par train ou } \\
\text { tramway }\end{array}$ & $25-30$ & Périurbain & Coût \\
\hline 1980 & Association Satorail & $\begin{array}{l}\text { Brotteaux-Crémieu } \\
\text { par SNCF ou CFEL }\end{array}$ & $16^{\prime}$ & $\begin{array}{l}\text { Périurbain } \\
\text { et intercités }\end{array}$ & $\begin{array}{l}\text { Surdimensionné et } \\
\text { coût }\end{array}$ \\
\hline 1987 & SNCF & $\begin{array}{l}\text { Trains par la ligne du } \\
\text { sud (Vénissieux) }\end{array}$ & $25^{\prime}$ & Régional & Nœud ferroviaire \\
\hline 1988 & Satorail & $\begin{array}{l}\text { CFEL avec trains de } \\
\text { banlieue ou Eurexpo } \\
\text { (LER) }\end{array}$ & $25-30^{\prime}$ & Périurbain & Non rentable \\
\hline 1989 & SYTRAL, SEMALY & $\begin{array}{l}\text { LRT Métro léger } \\
\text { Mégabus } \\
\text { Bus guidés } \\
\text { Tramway }\end{array}$ & $\begin{array}{c}25^{\prime} \\
22-24^{\prime} \\
25^{\prime} \\
17^{\prime}\end{array}$ & $\begin{array}{l}\text { Périurbain } \\
\text { Eurexpo } \\
\text { Eurexpo } \\
\text { Périurbain }\end{array}$ & $\begin{array}{c}\text { Pas de connexion } \\
\text { régionale, matériel } \\
\text { Financement }\end{array}$ \\
\hline 1994 & $\begin{array}{l}\text { Tramel } 2000 \\
\text { Conseil général du } \\
\text { Rhône }\end{array}$ & $\begin{array}{l}\text { Tramway express sur } \\
\text { CFEL }\end{array}$ & $40^{\prime}$ & Périurbain & $\begin{array}{l}\text { Elections } \\
\text { municipales }\end{array}$ \\
\hline 1996 & $\begin{array}{l}\text { Région Rhône-Alpes } \\
\text { Agence d'Urbanisme }\end{array}$ & $\begin{array}{l}\text { TERGV St-Etienne - } \\
\text { Grenoble }\end{array}$ & & Régional & $\begin{array}{l}\text { Nœud ferroviaire } \\
\text { Long terme }\end{array}$ \\
\hline 2001 & CFEL & Tramway & $20^{\prime}$ & Périurbain & 2008-2011 \\
\hline
\end{tabular}

Doc.5b - Historique des projets de liaison Lyon - Satolas/Saint-Exupéry (carte)

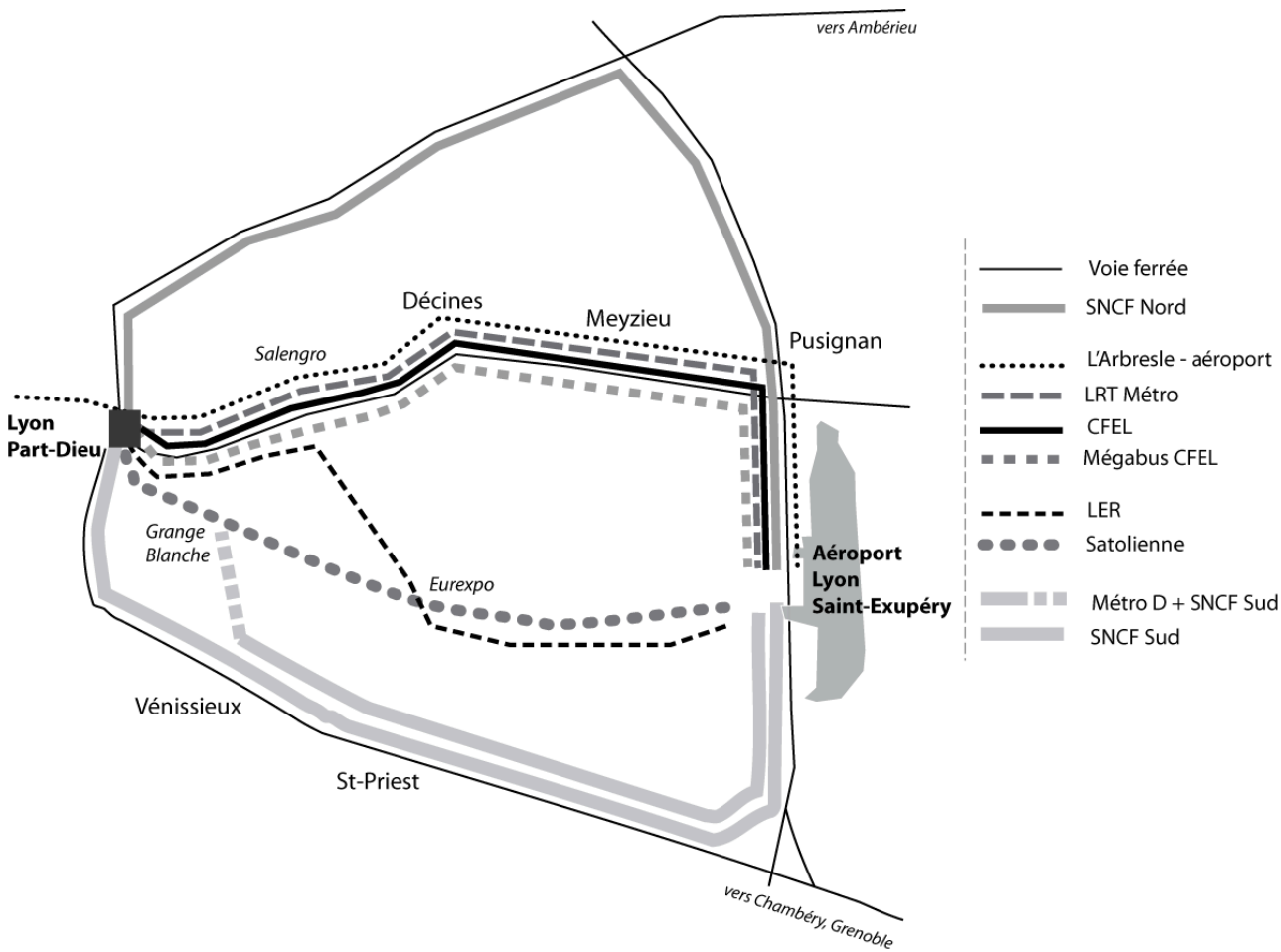

D'un côté, dans un contexte de croissance urbaine lyonnaise soutenue, l'extension de l'agglomération, orientée depuis plus d'un siècle en direction de l'Est et du Sud-Est, se poursuit préférentiellement dans cette même direction, d'abord dans ses banlieues puis 
dans les communes situées au-delà, qui entrent dans ce qui devient progressivement le milieu périurbain. Dans cette "ruée vers l'Est ", le secteur situé entre les axes LyonAmbérieu-Genève au nord et Lyon-Grenoble au sud devient une terre d'accueil de grands équipements (ville nouvelle, aéroport...) pendant la décennie 1966-1975, connaît ensuite une décennie d'essor anarchique avant d'être considéré, à partir de 1985, comme un espace stratégique (Montès, 2003).

Ce secteur, au milieu duquel se trouve l'aéroport, souffre alors à la fois d'un souséquipement en voirie urbaine adaptée à cette nouvelle croissance et d'une desserte très insuffisante en transports en commun par rapport aux besoins croissants. Au point que les territoires concernés, mal desservis, réclament de façon urgente un désenclavement significatif, clameur qui se fait de plus en plus intense au cours des décennies 1990 et 2000.

D'un autre côté, l'essor du trafic de l'aéroport obère peu à peu l'argument d'insuffisance jusque-là brandi pour justifier une desserte ferroviaire de l'aéroport. En effet, celui-ci double en vingt ans (3,7 Mp. en 1990, 6 Mp. en 2001 et 8 Mp. En 2010). Même si la vocation régionale de l'aéroport se fait davantage prégnante, le nombre potentiel de clients cheminant entre l'aéroport et le centre-ville croît jusqu'à approcher la valeur souhaitée.

Enfin, avec le temps, les esprits aussi évoluent, les logiques de cloisonnement institutionnel font progressivement place à des logiques de coopération. Ils montrent un élargissement des horizons et envisagent désormais une meilleure intégration des possibilités techniques, économiques et juridiques au regard des objectifs, des moyens et des opportunités, le tout dans un contexte d'urgence d'amélioration des dessertes. Un assouplissement des positions des acteurs et la marche vers une coopération institutionnelle entre AOT et entre opérateurs de réseau font leur chemin.

A ces évolutions lentes s'ajoutent des évolutions rapides, notamment dans la décennie 2000 , sur les plans politique et réglementaire.

49 Tout d'abord, le changement idéologique de la décennie 1990 qui met en place la notion même de PDU ${ }^{5}$ débouche, à Lyon, sur l'irruption du tramway (lignes T1 et T2), lequel va jouer un rôle majeur dans l'évolution du processus concernant la desserte de l'Est lyonnais. Partant du principe qu'il est moins compliqué d'ajouter un segment à un réseau existant que de créer un réseau de toutes pièces, il paraît alors logique d'utiliser ce tramway nouveau, surtout de la part du SYTRAL favorable de longue date à cette option de desserte périurbaine par un transport en commun lourd et moderne.

Ensuite, la marche progressive vers la déréglementation dans les transports en Europe à la fin des années 1980 atteint, vingt ans plus tard, le transport ferroviaire de voyageurs. A la suite de la partition du système ferroviaire français, en 1997, en deux entités (EPIC): l'une gestionnaire du réseau d'infrastructures (RFF) et l'autre exploitante de réseau (SNCF), l'introduction, sur ce segment du marché, de la libéralisation et de l'ouverture à des opérateurs privés va faire émerger des perspectives jusque-là impossibles à imaginer.

Venant en appui supplémentaire, l'essor des partenariats public-privé (PPP) va faciliter les choix et débloquer des processus.

Si les différents acteurs territoriaux majeurs (Grand Lyon, Conseil général du Rhône, Chambre de Commerce et d'Industrie, Région) ont contribué à la réflexion générale, à la 
conduite d'études et à l'avancement du processus, c'est bien le Conseil général du Rhône qui devient l'acteur fédérateur, le coordonnateur et l'initiateur de décisions fortes, en partenariat avec le SYTRAL. Le rôle primordial a bien été exercé par l'échelle locale, celle de l'agglomération lyonnaise et du département du Rhône, et non pas par l'échelle régionale.

Après le vote d'un projet élaboré conjointement par un groupement Conseil général Grand Lyon - SYTRAL (22 juin 1998), le Conseil général du Rhône adopte le 25 février 2001 le principe d'une réutilisation de la voie ferrée CFEL, modernisée, pour assurer du transport de voyageurs par un tramway urbain jusqu'à Meyzieu ZI, avec transformation de la plate-forme ferroviaire en ligne tramway. Il ajoute une connexion avec le métro (ligne A), prolongé de la station Laurent Bonnevay à la future station tramway de Vaulx-en-Velin - La Soie, futur pôle d'échanges et future porte orientale du centre de Lyon. La desserte urbaine et périurbaine l'emporte; néanmoins, le Conseil général exprime en outre un avis favorable à une extension ultérieure de la ligne jusqu'à l'aéroport. Cinq ans plus tard, en 2006, la ligne T3 des TCL est ouverte de Lyon-PartDieu à Meyzieu ZI et le désenclavement de l'Est lyonnais par une infrastructure lourde de transport est réalisé.

Deux hommes auront œuvré pour mettre un terme à la période d'hésitations et pour enclencher un processus de décision qui intègre les deux options initiales: Gérard Collomb, Président du Grand Lyon, et Michel Mercier, Président du Conseil général du Rhône.

Ce dernier, également député du Rhône, débloqua la situation en ce qui concerne l'extension jusqu'à l'aéroport, en faisant voter par l'Assemblée Nationale - dans un contexte de déréglementation - un amendement à la LOTI autorisant un Département à devenir AOT ferroviaire hors des limites d'une agglomération (2004). Dès lors, plus aucun obstacle ne se dresse pour desservir l'aéroport. Le Conseil général du Rhône, devenant AOT ferroviaire, définit un cahier des charges, lance un appel d'offres, ouvert au secteur privé par suite de la libéralisation du transport ferroviaire de voyageurs, choisit le concessionnaire (15 décembre 2008), la compagnie Rhônexpress, qui en moins de deux ans achève le chantier et ouvre la ligne Rhônexpress ( 9 août 2010). En outre, à la fois concédant de cette ligne gare-aéroport et partenaire du SYTRAL (T3), le Conseil général du Rhône définit un cahier des charges de bonne entente entre les deux opérateurs de réseau retenus sur cette seule et unique infrastructure. On ne manquera pas de relever le poids de ce territoire dans la dénomination de ce tramway, l'appellation Rhônexpress mettant en avant l'AOT départementale, et de souligner alors l'évidente absence de visibilité de la métropole lyonnaise, vue de l'extérieur.

Si les deux types de desserte sont finalement offerts, il apparaît clairement que l'agglomération lyonnaise aura été un (f)acteur fondamental: sans son extension progressive vers l'Est, sans le développement d'un réseau de tramways en ville, il n'y aurait probablement toujours pas de desserte aéroportuaire, sans déréglementations successives non plus. Au temps long de concertation entre acteurs a fait suite un temps assez rapide de prises de décisions réussissant à combiner les deux formules et à assurer la mixité fonctionnelle. Dans ce processus, les logiques locales ont prévalu sur les logiques nationales. 


\section{III . L'intégration multi-territoriale}

la question est aussi de savoir si celles-ci sont traitées de façon isolée, voire indépendante du reste, ou si elles sont considérées plus globalement comme des éléments d'un réseau plus vaste. En l'occurrence, leur intégration dans un réseau de transports en commun est-elle réalisée ? et ces dessertes contribuent-elles à intégrer les territoires concernés?

$\mathrm{Au}$ cœur de la réponse, l'intermodalité tient évidemment une grande place; en la matière et dans le strict contexte français, Lyon est une agglomération en pointe et au premier rang, sans pour autant atteindre le niveau des métropoles de l'Europe rhénane.

\section{A. Une intégration par la multiplication de pôles d'échanges}

Dans la suite des politiques de transports en commun à Lyon depuis trois décennies, qui privilégient une logique de réseau plutôt qu'une logique de tronçons et une logique de déplacements plutôt qu'une logique d'entreprise, de multiples connexions entre modes de transport ont été réalisées.

61 L'intermodalité est réalisée ponctuellement en plusieurs lieux égrenés le long de la ligne de l'Est lyonnais.

D'une part, dans six stations intermédiaires sur huit, la présence d'un parc-relais souligne la volonté de favoriser le report modal en rabattant des automobiles individuelles vers des transports collectifs tout en incluant le coût du stationnement dans le billet de ces derniers. Parmi ces six stations, cinq ont été dotées de parcs à vélos, cinq d'un pôle bus et une d'une station de métro par suite du prolongement de la ligne A de Laurent Bonnevay à La Soie.

Doc.6 - Les équipements des pôles d'échange le long du T3

\begin{tabular}{|c|c|c|c|c|c|c|}
\hline & Station tramway T3 & $\begin{array}{l}\text { Parking } \\
\text { (places) }\end{array}$ & $P+R$ & $\begin{array}{l}\text { Pôle } \\
\text { bus }\end{array}$ & $\begin{array}{l}\text { Parc } \\
\text { à } \\
\text { vélos }\end{array}$ & Métro \\
\hline \multirow{6}{*}{ Ouest } & Gare de Villeurbanne & 70 & & & & \\
\hline & Vaulx-en-Velin / La Soie & 300 & & & & \\
\hline & Décines - Centre & 110 & & & & \\
\hline & Décines - Grand Large & 70 & & & & \\
\hline & Meyzieu Gare & 110 & & & & \\
\hline & Meyzieu ZI & 500 & & & & \\
\hline & Total & 1160 & & & & \\
\hline
\end{tabular}

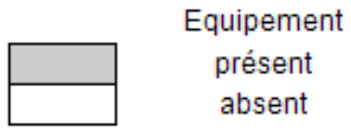

Ainsi un pôle d'échanges complet (tramway, bus, métro, vélo, $\mathrm{P}+\mathrm{R}$ ) a émergé dans la banlieue Est de Lyon en sus de celui de Laurent Bonnevay, à La Soie; et deux autres pôles d'échanges, Grand Large et Meyzieu ZI, combinent les bus et véhicules légers avec 
le tramway. Si la station de La Soie est une porte de la ville située en banlieue, celle de Meyzieu ZI est une porte périurbaine.

64 A ces parkings aménagés par le SYTRAL va s'ajouter un parking Rhônexpress, réservé aux clients aériens et aux clients TGV de Part-Dieu (prix intégré dans le tarif tramway), avec le transfert de la gestion du Parking de La Soie du SYTRAL à Rhônexpress ; l'idée germe aussi d'attirer ici également des clients des parkings du centre de Lyon.

D'autre part, aux deux extrémités de la ligne Rhônexpress, l'intermodalité est également réalisée.

A l'aéroport, celle-ci s'effectue à la gare et non pas à l'aérogare ; la station du tramway Rhônexpress a été implantée dans la gare TGV au même niveau que les voies à grande vitesse, du côté occidental, à un emplacement réservé vingt ans auparavant lors de la construction de la gare TGV (1990-94). Cette plate-forme ferroviaire connecte désormais une desserte TGV, un tramway et le véhicule léger (parkings P5 et P6) ; elle est reliée à l'aérogare depuis sa création par une passerelle dotée d'un tapis roulant en raison de la distance. L'essentiel est donc bien l'ajout d'une liaison ferroviaire cadencée entre l'aéroport et le centre-ville.

67 A la gare centrale de la Part-Dieu, l'adjonction des lignes T3 et Rhônexpress renforce notablement la nodalité du site, en quantité et en qualité, ainsi que l'intermodalité du lieu, en particulier en connectant mieux les échelles de mobilité, périurbaine et banlieusarde, urbaine et nationale.

Toutefois à très grande échelle, celle de la gare, l'intermodalité est péjorée de deux manières. Tout d'abord, le débranchement de la ligne en gare de la Part-Dieu, où la voie CFEL arrivait, et son rejet à l'extérieur de la gare du côté de Villeurbanne (Est), dans la voirie urbaine - par suite des besoins d'ajouter une voie et un quai pour faire face à la saturation des voies et à la réorganisation des circulations en gare - ont fait disparaître au moins ici l'éventualité d'un futur tram-train. Les lignes T3 et Rhônexpress sont des tramways circulant sur une ancienne voie ferrée où désormais seuls circulent des tramways.

69 Par ailleurs, une chaîne de transports entre le périurbain oriental, le centre-ville ou d'autres quartiers de Lyon via le réseau de tramways lyonnais souffre d'une nette rupture de charge entre les lignes T3 et T1, avec la longue traversée obligée du hall de gare puis du parvis de la gare dans un cheminement piétonnier à risques aux heures de pointe (bondé).

70 En sus des intermodalités ponctuelles, peut-on imaginer une intermodalité linéaire? Potentiellement oui en cas de connexions éventuelles des lignes de l'Est lyonnais par exemple avec celles des réseaux de l'Ouest lyonnais via la gare Saint-Paul, où est actuellement mis en place un réseau de tram-trains. Ce qui autoriserait alors une liaison tram-train entre l'Ouest lyonnais et l'aéroport, mais avec quel(s) opérateur(s) de réseau(x) (TCL, Rhônexpress, SNCF...) ? avec quelle(s) rupture(s) de charge ou non? et avec quelle(s) durée(s) de trajet?

71 Ceci étant et malgré ces manques, l'intégration de la ligne de l'Est lyonnais dans un système de transport multimodal et intermodal est réalisée, impliquant logiquement une intégration des territoires banlieusards et périurbains ainsi que de l'aéroport au territoire de la grande agglomération lyonnaise. 


\section{B. Une intermodalité régionale aéroportuaire délaissée}

72 Au-delà, par comparaison avec les autres métropoles européennes, la réflexion ne peut faire l'économie d'une interrogation sur l'application au cas lyonnais du modèle de trinôme d'interconnexion (Varlet, 1992).

73 Tout en étant l'un des premiers aéroports européens à accueillir la grande vitesse ferroviaire l'aéroport de Lyon souffrait de deux maux : d'une part un accès de la gare exclusivement réservé aux TGV, sans jamais autoriser le passage et l'arrêt de trains régionaux, et d'autre part l'absence d'une liaison ferroviaire dédiée entre les deux plates-formes. Aujourd'hui, avec cette liaison Rhônexpress, rapide, fréquente, confortable et à l'intermodalité efficace, ce deuxième manque est supprimé.

74 Ceci étant, l'attribution de la voie ferrée aéroport - centre-ville à un tramway, débranché du réseau ferré à Part-Dieu de surcroît, à deux opérateurs spécifiques, et dédiée à des liaisons aéroport - centre-ville, élimine de facto une interconnexion régionale à l'aéroport. Si l'intermodalité est effective entre l'avion et l'automobile depuis l'origine et que l'aéroport est bien accessible grâce à un dense réseau autoroutier, si elle l'est aussi entre TGV et avion - toutefois bien contrariée par la noncorrespondance entre les deux modes - l'interconnexion ferroviaire régionale est absente. Celle-ci aurait pu se traduire par la desserte successive de l'aéroport et de Part-Dieu par des trains régionaux, mais la SNCF a toujours refusé d'accueillir des trains régionaux à Satolas, malgré les perspectives initiales de la Région et contrairement à beaucoup d'autres aéroports européens. Le choix de l'agglomération lyonnaise d'opter pour une desserte tramway réduit, de fait, la dimension régionale potentielle de l'aéroport Saint-Exupéry, qui l'est pourtant bien en termes d'intermodalité voiture/avion mais qui ne le sera pas entre le train et l'avion.

\section{Conclusion}

Pionniers en France, les tramways de l'Est lyonnais le sont : sur le plan des circulations en tant que première voie ferrée régionale à être ouverte à deux tramways, l'un urbain et l'autre pour desservir l'aéroport d'une métropole régionale ; sur le plan juridique car la première à être régie par deux AOT et à être confiée à deux opérateurs différents, l'un public et l'autre privé, avec également le premier exemple de partenariat publicprivé ferroviaire; et sur le plan technique avec deux offres très contrastées et coordonnées sur une même voie pour desservir deux clientèles bien différentes.

Ils correspondent à un compromis autorisant les deux types de dessertes, sans toutefois permettre une desserte régionale via les deux plates-formes, compromis rendu possible par les évolutions juridiques (déréglementations diverses), par les évolutions du trafic aérien et de l'extension de l'agglomération, et par le rôle croissant puis fondamental du Conseil général du Rhône dans la résolution du problème et dans l'avancement du dossier.

Si le problème de la desserte ferroviaire de l'aéroport fut l'argument initial et permanent pendant des décennies et se posa en termes de tracés et de modes de transport, il a été le dernier à être résolu par rapport au problème de desserte (péri)urbaine considéré plus tard comme plus urgent. L'agglomération lyonnaise a donc fait prévaloir d'abord sa desserte interne plutôt que sa connexion avec un lieu tremplin 
vers l'international, avant de favoriser cette dernière. Un exercice laborieux qui aura tout de même nécessité près de quatre décennies... ce qui, là encore, constitue une originalité puisqu'une très longue phase de réflexion aura précédé les réalisations, évitant d'entamer des chantiers épars et étalés dans le temps, souvent synonymes d'aménagements irréparables. Au reproche du temps long de la concertation, on opposera l'idée que, dans une ambiance de discordance entre territoires fonctionnels et territoires institutionnels, les uns et les autres des acteurs seront peu à peu parvenus à les accorder.

\section{BIBLIOGRAPHIE}

BONNEVILLE M. (1997), Lyon, métropole régionale ou Eurocité?, Paris, Anthropos, 221p.

MATHURIN A. (2002), « Le trinôme d'interconnexion lyonnais », Mémoire Maîtrise de Géographie, Université de Toulouse-Le Mirail, $180 \mathrm{p}$.

MONTÈS C. (2003), Les transports dans l'aménagement urbain à Lyon, Collection Géocarrefour, 264 p.

PLASSARD F. (1990), « Le devenir de Satolas », Revue de Géographie de Lyon, vol. 65 n¹, pp. 52-54.

S.E.P.A.L. (1988), Lyon 2010. Un projet d'agglomération pour une métropole européenne, Lyon, 194 p.

VARLET J. (1992),L'interconnexion des réseaux de transport en Europe, Institut du Transport Aérien (ITA), Études et documents, vol. 24, 1992/1, Paris, 198 p.

VARLET J., (2000), Dynamique des interconnexions des réseaux de transports rapides en Europe : devenir et diffusion spatiale d'un concept géographique, Flux, $\mathrm{n}^{\circ}$ 41, pp. 5-16.

VILLE ET TRANSPORTS Magazine (2006), Lyon, un modèle du transport urbain français ?, n412, 22 novembre, pp. $28-48$

\section{NOTES}

1. Loi sur l'air et sur l'utilisation rationnelle de l'énergie, 30 décembre 1996.

2. Loi relative à la Solidarité et au Renouvellement Urbains, 13 décembre 2000.

3. Grenelle de l'Environnement, 23 juin 2009.

4. Autorité Organisatrice de Transports Urbains

5. Plan de Déplacements Urbains. Inscrit dans la LOTI (1982), il devient obligatoire avec la LAURE (1996) et est renforcé par la Loi SRU (2000). 


\section{RÉSUMÉS}

Malgré son statut d'étude de cas, l'analyse des tramways de l'Est lyonnais ne manque pas d'intérêt sur le plan général. Elle révèle de notables transformations dans le transport de voyageurs au sein d'une grande agglomération: les difficultés des territoires à satisfaire des logiques de desserte contraires ou peu conciliables (banlieue, périurbain, aéroport), le rôle de l'évolution des contextes généraux (extension urbaine, déréglementations successives) dans l'adaptation des solutions locales et dans la combinaison des formules envisagées, les aspects innovants et originaux du dispositif adopté. Sur une même infrastructure ferroviaire circulent désormais deux tramways différents, relevant de deux types d'opérateurs (public, privé) et de deux autorités organisatrices de transports qui assurent deux types de dessertes, la ligne étant intégrée dans le système de transport multimodal et intermodal de l'agglomération lyonnaise mais pas dans un système de desserte régionale.

\section{INDEX}

Mots-clés : tramway, transports urbains, intermodalité, aéroport, périurbain

\section{AUTEUR}

\section{JEAN VARLET}

UMR 5204 EDYTEM CNRS-Université de Savoie - Pôle Montagne, Campus scientifique - 73376 Le Bourget-du-Lac cedex. jean.varlet[chez]univ-savoie.fr 